\title{
Fish Cam: An Online Tool for Introducing Shoaling Behavior to the Classroom
}

\author{
Maura Southwell, Maria Galassi, and Scott McRobert
}

\begin{abstract}
Fish Cam is an on-line educational resource that enables students to participate in behavioral research projects without ever leaving their classroom. By linking onto the Fish Cam site, students will observe an experimental tank in which fish choose shoal-mates in dichotomous choice tests. In these experiments, a test fish, in the central compartment, displays its shoaling preference by swimming near small shoals of fish in either of two side compartments. Assays are designed to examine the effects of phenotype, shoal size, and other factors known to influence shoaling. Students monitor Fish Cam in real time, and students collect data simply by running timers when the test fish crosses into the preference zones at each end of the central compartment. The times are logged onto data sheets that we provide, and we assist the students with their analysis. The simplicity of shoaling behavior makes it an ideal model system for data collection that is accessible to students of all ages and, in its first few years of operation, Fish Cam studies have been performed by fifth-, seventh- and eleventh-grade students. Sample lesson plans and handouts are available online to enhance the Fish Cam experience. The ultimate goals of this project are to make scientific research accessible in the classroom and promote science education.
\end{abstract}

\section{Introduction}

$S^{\text {s }}$ HOALING BEHAVIOR is a common component in the life history of many species of fish and provides a wonderful model system for collecting and analyzing data. Our laboratory has been studying shoaling behavior for years, and in this article we describe our latest project, Fish Cam, an on-line resource for educators to incorporate hands-on learning in their lessons. Fish Cam shows an experimental shoaling tank to which we add test fish and target fish regularly, enabling teachers and students to collect data along with us without ever leaving their classrooms. Here we explain shoaling behavior, along with the simple methodology for collecting shoaling data in the hopes that Fish Cam will become a widespread opportunity for providing students with experience in scientific method, experimental design, data collection, and analysis.

For most organisms, a specific term is used to describe social groupings. Whales swim in pods, wolves run in packs, and birds fly in flocks. What about fish? Ask most people to name a group of fish and they will almost always say "school." However, this term is not always correctly used. To be considered a school, a group of fish must display complex synchronized behavior, swimming in the same direction, at the same speed, and turning simultaneously. ${ }^{1}$ The word shoal, on the other hand, is the proper term when describing any simple social grouping of fish. In short, any group of fish is a shoal, but not all shoals demonstrate the properties of a school. Similarly, while most fish appear to shoal, not all fish school. ${ }^{2-4}$

The fact that shoaling appears to be a common behavior among many different species of fish suggests this behavior must offer distinct evolutionary advantages. These advantages appear to include increased success in finding food, access to potential mates, and increased protection from predators. According to the "many eyes phenomenon," fish in shoals find food faster and, consequently, spend more time feeding rather than foraging. This concept is derived from the simple fact that large groups of fish have been shown to have greater success finding food than smaller groups or single individuals. ${ }^{5}$ Furthermore, within a larger shoal, the opportunity for conspecific mating greatly increases by bringing males and females into close proximity with each other. The anti-predatory benefits, which have been the most extensively studied, are described as three main phenomena: the "numerical dilution effect," the "confusion effect," and the "oddity effect." The numerical dilution effect relies on the statistical idea that being part of a larger shoal reduces the chance of being killed. For example, in a shoal of 100 fish, each individual has only a one-in-one-hundred chance of being targeted by a predator. The other two phenomena are very

Department of Biology, Saint Joseph's University, Philadelphia, Pennsylvania. 
closely related and rely heavily on the phenotypic similarities between the individuals in the shoal. The confusion effect suggests that a predator, confronted with a large group of similar-looking individuals, experiences perceptual confusion and has difficulty identifying a single individual fish to attack. ${ }^{6-9}$ Conversely, the oddity effect describes how an individual fish that does not resemble the other members of the shoal will be more likely to attract a predator's attention. ${ }^{10}$ Not surprisingly, most fish appear to be very good at choosing shoalmates, using features such as body size, ${ }^{11}$ striping pattern, ${ }^{12}$ and body coloration ${ }^{13}$ to shoal with fish displaying similar characteristics to themselves.

In our laboratory, we have examined shoaling behavior in a wide variety of species, including mollies (Poecilia latipinna), guppies (Poecilia reticulata), fighting fish (Betta splendens), and even bluegills (Lepomis macroschirus) and rock bass (Ambloplites rupestris). However, owing to their popularity and widespread use as a model organism, the zebrafish (Danio rerio) is an ideal species to explore the factors that influence shoaling decisions. With transparent embryos and larvae, relatively quick life cycles, and large numbers of offspring per spawning, zebrafish offer a variety of options for genetic, physiological, developmental, and neurological studies. What's more, a recent surge in research focusing on their ecology and behavior ${ }^{14}$ has led to increased interest in the basic biology of this species. From our point of view, zebrafish are active swimmers and show a strong propensity to shoal under experimental conditions. ${ }^{15}$ In our laboratory, we have studied a number of different aspects to zebrafish shoaling, including the interaction between $D$. rerio and the related pearl danio $\left(D\right.$. albolineata), ${ }^{16}$ the shoaling behavior of the transgenic "Glofish,"17 and even the possibility that shoaling behavior follows a circadian pattern. ${ }^{18}$ Each of these studies, and many more from other laboratories, provides a strong foundation of literature on which to build future experiments, and encourage the involvement of future scientists.

Launched in the spring of 2010, Fish Cam is an on-line website intended to reach out to the surrounding community and introduce students and teachers to behavioral biology and research. During the Family Science Days program at the
2011 American Association for the Advancement of Science (AAAS) annual conference in Washington, D.C., teachers, students, and scientists alike were able to observe Fish Cam in action and record their own data via a live web feed coming from the Biodiversity Laboratories in Philadelphia, PA. Those who attended the conference were encouraged to contact the lab directly to plan and organize personalized experiments to fit the students' educational goals. Because the study of shoaling behavior is relatively simple to understand, this research technique can be applied to a wide range of educational levels by altering the complexity of each experiment.

\section{Materials and Methods}

Fish Cam is located in one of the rooms of the Biodiversity Laboratories at Saint Joseph's University and consists of two Linksys internet monitoring cameras, each focused on its own experimental shoaling tank (Fig. 1). Holding tanks nearby house the fish being used in each experiment. The fish are typically obtained from a local importer who supplies fish for the pet trade, although for specific studies fish might be ordered from other sources such as a zebrafish stock center. All fish are fed daily (food choice depends on species, although we typically use a commercial food sold in pet shops, e.g., "TetraMin (C Tropical Flakes") and the test tanks and holding tanks are maintained at species-appropriate temperatures with the lights running on a 12:12 LD cycle. The methodologies involved in the acquisition, care, and study of fish used in Fish Cam have been approved by the Saint Joseph's University IACUC.

The test tanks are 20 -gallon aquariums $\left(30^{\prime \prime} \times 12^{\prime \prime} \times 12^{\prime \prime}\right) \mathrm{di}-$ vided into thirds by the addition of two panes of glass, $7.5^{\prime \prime}$ from each end wall, creating two small end compartments $\left(7.5^{\prime \prime} \times 12^{\prime \prime} \times 12^{\prime \prime}\right)$ and a large central compartment $\left(15^{\prime \prime} \times 12^{\prime \prime} \times 12^{\prime \prime}-\right.$ Fig. 2$)$ with filters, heaters, and gravel substrate. "Target" shoals (e.g., a small group of zebrafish or tetras) are placed into the end chambers and an individual test fish is added to the central chamber. During an experiment (typically 600 seconds in length), the time the test fish spends within preference zones, 2" from each end chamber is

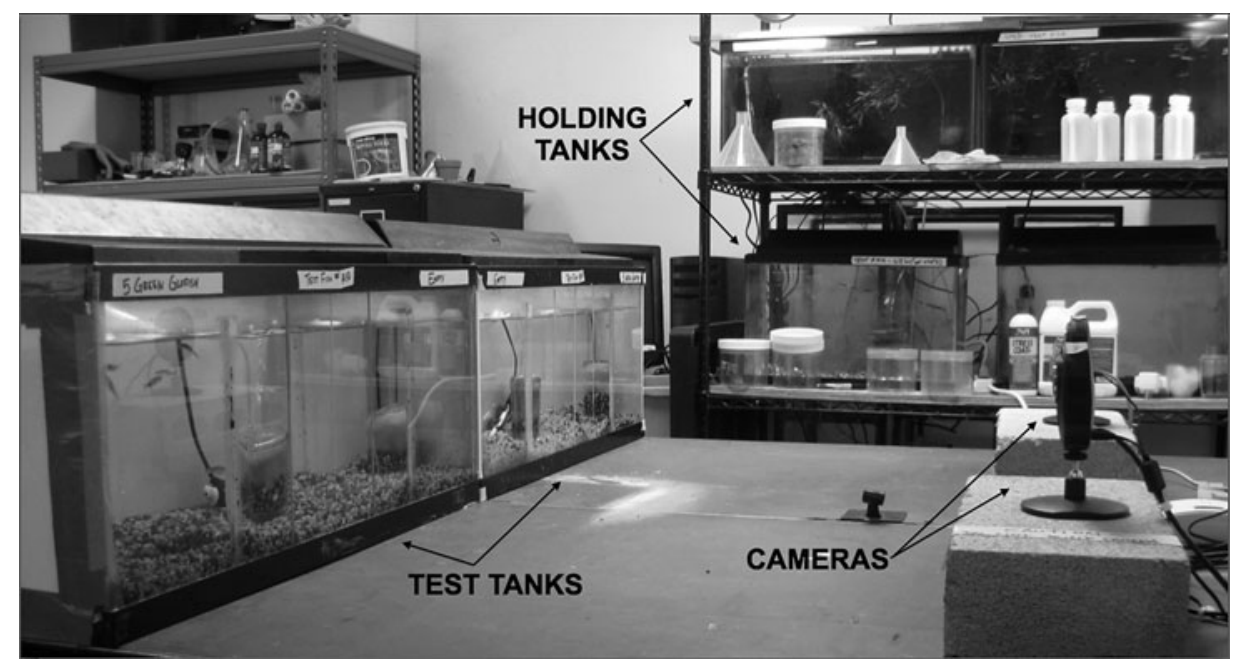

FIG. 1. The Fish Cam set-up: Two Linksys internet cameras, each focused on its own experimental shoaling tank. Holding tanks for experimental fish can be seen in the background. 


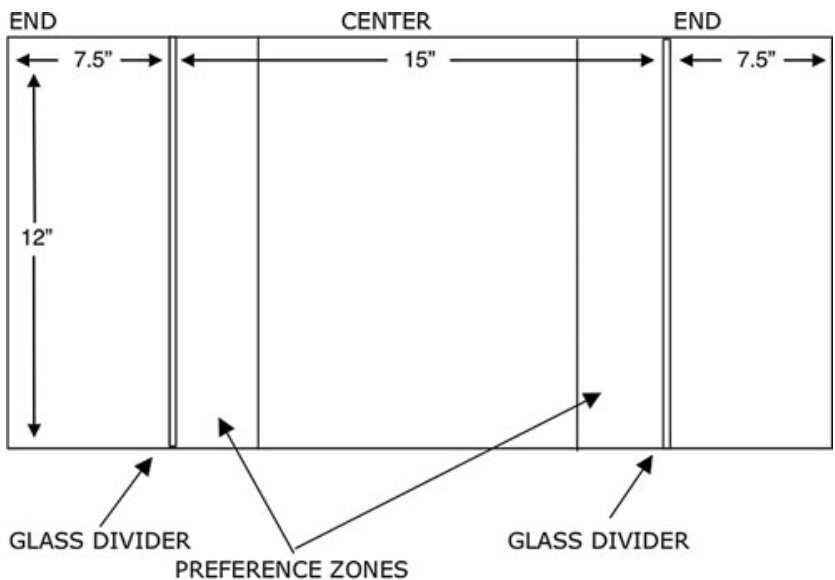

FIG. 2. Diagram of experimental tank for shoaling studies.

recorded. Sample sizes are typically 20 test fish for each assay, and each test fish is used only once, unless a specific experiment is designed around the effects of experience. Differences in the amount of time spent by test fish in each preference zone are compared using the statistical program SPSS to run a paired $t$-test. Significant differences (at $p<0.05$ ) indicate a preference for the shoal in one of the end chambers. We suggest that teachers explain the value and concepts underlying statistical analyses to their students, as appropriate to age level, and we assist in a discussion on how $p$ values relate to the observed behaviors.

\section{Results}

During Fish Cam studies (following the experimental procedure outlined above), we set up the test tanks, placing the target shoals in the end compartments, and add new test fish according to a schedule that we develop with the participating teachers and students. Sometimes we add a new test fish daily, sometimes we add new test fish many times during a day. Teachers and students monitoring Fish Cam will need two stopwatches to record the amount of time the test fish spends in each preference zone and a clock to monitor the $600 \mathrm{sec}$ observation period. Data can be entered onto the worksheets (see Supplementary Materials; Supplementary data are available online at www.liebertpub.com/zeb) and available at our website (<http://www.sju.edu/academics/ cas/biology/resources/biodiversity/fishcam/index.html > http://www.sju.edu/academics/cas/biology/resources / biodiversity/fishcam/index.html).

A sample experiment might involve a series of tests. First, asking the test fish to choose between a small shoal of conspecifics and an empty chamber to determine whether these fish demonstrate basic shoaling behavior. Second, asking the test fish to choose between a small shoal of non-conspecifics and an empty chamber to see if they will spend time near another group of fish when that is the only chance to shoal. Third, asking test fish to choose between a small group of conspecifics and a small group of non-conspecifics to see whether they can discriminate between shoalmates on the basis of species composition.

Teachers and students interested in Fish Cam can visit our website (at < http://www.sju.edu/academics/cas/biology/ resources/biodiversity/fishcam/index.html > http://www .sju.edu/academics/cas/biology/resources/biodiversity/ fishcam/index.html). Here they find a Brochure (Supplementary Material 1), along with a set of generalized Lesson Plans (Supplementary Material 2) for students in grades 5-8, plus a Pre-Lab Handout (Supplementary Material 3); a Fish Cam Procedure Handout (Supplementary Material 4); an Observation Worksheet (Supplementary Material 5); and a simple Fish Anatomy Worksheet (Supplementary Material 6). The Pre-Lab Worksheet, which can be completed either during or outside of class, encourages students to search online for important concepts they will apply during the experiment. This handout requires the students to define vocabulary terms and research background information on camouflage and fish behavior in the wild. The Procedure and Observation Worksheets can be used in the classroom to emphasize the scientific method. The Observation Worksheet asks questions that the students must consider when developing their hypotheses and drawing conclusions from the shoaling assay, while the Procedure Worksheet focuses on the execution of the experiment. We work with the teacher and class to develop a plan for a shoaling study and are always ready to make alterations in the study as may be needed. In this way, Fish Cam is more than a simple delivery system, but rather a system that can be changed throughout the experiment as the teacher and class develop new needs, which are communicated to us through email directed to the director of the Biodiversity Lab. Generally, the first testing session is encouraged to take place during the class period so students will have a chance to clearly understand the procedural techniques and have the opportunity to ask any questions that may arise. The remaining assays can then be carried-out by the students individually, outside of the classroom, as homework. The total number of assays to be observed will vary with the study, but might run something like this: Three separate assays could be run over a period of 3 weeks with two assays being observed/ day and students being assigned specific times to observe the tank. This would enable the class to collect thirty data points over the 3-week period.

As an example of the work that can be performed using Fish Cam, we ran an experiment during the spring of 2012 to determine the effect of body coloration on shoalmate choice in zebrafish (D. rerio). In this study, wild-type (wt.) zebrafish (obtained from the pet trade) were given one of three shoaling choices: Assay 1) a shoal of five wt. zebrafish vs. an empty

Table 1. Mean Association Time \pm SE Exhibited by Wild-Type Aebrafish in Shoalmate Choice Tests

\begin{tabular}{|c|c|c|c|c|c|c|}
\hline Assay & Shoal 1 & Mean time $(s) \pm S E$ & Shoal 2 & Mean time $(s) \pm S E$ & $\mathrm{t}$ & $\mathrm{p}$ \\
\hline 1 & $5 w t$ & $315.9 \pm 22.5$ & Empty & $115.5 \pm 17.5$ & 5.487 & $<0.001$ \\
\hline 2 & 5 Glofish & $381.5 \pm 30.4$ & Empty & $98.3 \pm 19.7$ & 5.966 & $<0.001$ \\
\hline 3 & $5 w t$ & $215.2 \pm 19.5$ & 5 Glofish & $228.8 \pm 22.9$ & 0.337 & 0.740 \\
\hline
\end{tabular}

$n=20$ for each behavioral choice test. Statistical analysis = paired $t$-test. 
compartment; Assay 2) a shoal of five green Glofish (obtained in the pet trade) vs. an empty compartment and; Assay 3) a shoal of five wt. zebrafish vs. a shoal of five Glofish.

The results showed that the wt. zebrafish performed standard shoaling behavior, spending significantly more time swimming near the group of wt. zebrafish than near the empty compartment, and significantly more time swimming near the group of Glofish than near the empty compartment. However, when given a choice between two shoals, there was no significant difference between the time spent swimming near the group of wt. zebrafish and the time spent swimming near the group of Glofish. These results suggest that the difference in coloration between wt. zebrafish and green Glofish is not sufficient to impact shoaling choices in D. rerio (results summarized in Table 1).

\section{Discussion}

Since the degree of complexity for shoaling experiments can greatly vary, the lesson plans for Fish Cam can easily change to fit the needs of the teachers and students. For example, high school students may incorporate a more detailed background research about specific fish species or perform their own statistical data analyses; whereas teachers of younger students may choose to focus on basic counting skills and color differentiations. Teachers are also encouraged to become directly involved in the Fish Cam project by donating any lesson plans, worksheets, and handouts, and activity procedures they develop throughout their experience. These contributions will be posted on the Fish Cam website and made available for other teachers to use in their classrooms. The feedback we receive from the participants allow us to greater understand the educational and professional needs of the teachers and students and improve the overall Fish Cam experience.

Fish Cam is an online educational resource for teachers and students who are interested in integrating real scientific research into their learning experience. Teachers can choose to design an entire lesson plan around Fish Cam with the available lesson plan worksheet and procedures or individual students can choose to work directly with us to design a unique science fair project. Through this resource, students will learn about the topic of animal behavior; discuss how to design, perform, and analyze scientific research; and utilize important skills including teamwork, organization, and time management. At the time of this writing, we have run experiments on basic shoaling behavior with students from $7^{\text {th }}$-, $8^{\text {th }}$-, and $11^{\text {th }}$ - grade, and hope that the future brings interactions with more students and their teachers. Now, as in the future, the goals of Fish Cam are to make real scientific inquiry available to students in their own classroom, to aid teachers in their quest to be great scientific educators, and to encourage a love of science in all students.

\section{Acknowledgments}

This work is supported by the Department of Biology at Saint Joseph's University, as well as grants from the Saint Joseph's University Chapter of Sigma Xi and a Howard Hughes Medical Institute Undergraduate Education Grant. We would like to thank the McNulty Scholars Program for Excellence in Science and Math. We would also like to thank two anonymous reviewers for their helpful comments, which contributed greatly to this manuscript.

\section{Disclosure Statement}

No competing financial interests exist.

\section{References}

1. Pitcher TJ. Heuristic definitions of fish shoaling behavior. An Behav 1983;31:611-613.

2. Krause J, Ruxton GD. Living in Groups. New York: Oxford University Press, 2002.

3. Brown C, Laland KN. Social learning in fishes: A review. Fish Fisheries 2003;4: 280-288.

4. McRobert SP. Shoaling behavior in fish. In: The Encyclopedia of Animal Behavior, Bekoff M, ed. Phoenix, AZ: Greenwood Press, 2004. Pp. 1012-1016.

5. Pitcher T, Magurran A, Winfield I. Fish in larger shoals find food faster. Behav Ecol Sociobiol 1982;10:149-151.

6. Neill SR, Cullen JM. Experiments on whether schooling of prey affects hunting behavior of cephalopods and fish predators. J Zool 1974;172:549-569.

7. Ohguchi O. Prey density and selection against oddity by three-spined sticklebacks. Zeitschrift Tierpsychol 1981;23:1-79.

8. Landeau L, Terborgh, J. Oddity and the 'confusion effect' in predation. An Behav 1986;34:1372-1380.

9. Magurran AE, Pitcher TJ. Provenance, shoal size and the sociobiology of predator evasion behaviour in minnow shoals. Proc R Soc Lond 1987;B229:439-465.

10. Theodorakis CW. Size segregation and the effects of oddity on predation risk in minnow schools. An Behav 1989;38:496502.

11. Croft DP, Arrowsmith BJ, Bielby J, Skinner K, White E, Couzin ID, et al. Mechanisms underlying shoal composition in the Trinidadian guppy, Poecilia reticulata. Oikos 2003;100: 429-438.

12. Rosenthal G, Ryan MJ. Assortative preferences for striped in danios. An Behav 2005;70:1063-1066.

13. McRobert SP, Bradner J. The influence of body coloration on shoaling preferences in fish. An Behav 1998;56:611-615.

14. Spence R, Gerlach G, Lawrence C, Smith C. The behaviour and ecology of the zebrafish, Danio rerio. Biol Rev 2008;83: 13-34.

15. Ruhl N, McRobert SP. The effect of sex and shoal size on shoaling behavior in the zebrafish (Danio rerio). J Fish Biol 2005; 67:1318-1326.

16. Snekser JL, Ruhl N, Bauer K, McRobert SP. The influence of sex and phenotype on shoaling decisions in zebrafish. Inter J Comp Psych 2010;23,70-81.

17. Snekser J, McRobert SP, Murphy CE, Clotfelter ED. Aggregation behavior in wildtype and transgenic zebrafish. Ethology 2006;112:181-187.

18. Paciorek T, McRobert SP. Daily variation in the shoaling behavior of zebrafish (Danio rerio). Cur Zoo-Special Edition: Ecol Evol Behav Fish 2012;58:129-137.

Address correspondence to: Scott McRobert

Department of Biology

Saint Joseph's University 5600 City Avenue

Philadelphia, PA 19131

E-mail: smcrober@sju.edu 